\title{
Chemotherapy in non-small cell lung cancer: time to re-examine our attitudes
}

Of 41000 deaths from lung cancer each year in the United Kingdom, 32000 are from non-small cell lung cancers (NSCLC). The prevailing attitude within the UK is that treatment of this disease is worthwhile if it is surgically resectable but, if not, palliative treatment should be offered. This usually involves using radiotherapy to treat local symptoms such as a painful metastasis, an obstructed bronchus, or superior vena cava obstruction. The recent contribution of the UK to clinical research in management of this most common cancer has been mainly to define radiotherapy doses for palliation ${ }^{1}$ and to explore hyperfractionated accelerated radiotherapy in the CHART trials. If we examine our national contribution objectively, it has been disappointingly unimaginative. The lead in the study of new approaches has come from North America and other European countries. ${ }^{23}$ Indeed, for a country of nearly 60 million inhabitants and one of the highest death rates from the disease in the western world, ${ }^{4}$ we have remarkably few groups undertaking clinical research, whether as early phase studies or large scale randomised trials.

The failure to originate new ideas in the UK is reflected in, and partly caused by, a climate of pessimism and nihilism among many pulmonary physicians and some oncologists. Damaging effects on clinical practice produced by some of the changes in the National Health Service have perhaps dealt a near mortal blow to those who would like to contribute to studies of novel treatment strategies in the disease. When it has become difficult to treat patients who benefit greatly from cancer treatment, why devote time and resources to a disease such as NSCLC?

This attitude is prevalent just at the time when some very interesting and encouraging new approaches are emerging. One of the most promising has been a redefinition of the possible role of chemotherapy in the disease. Trials of chemotherapy in NSCLC have been undertaken over the last 30 years and it is a remarkable, and not very creditable, reflection on the methodology of clinical trials during this time that it has taken so long to understand what is the likely contribution of this treatment. There are several reasons for this. Firstly, and most importantly, we have not always understood that, if chemotherapy benefits patients, the size of the benefit is likely to be quite small - perhaps only $5 \%$ or so. Trialists have often failed to grasp that the demonstration of such small differences would require very large scale studies. Perhaps we were not interested in differences of this size, but now we know that improvements in survival are likely to be of this order when chemotherapy is used in common cancers of adult life such as breast cancer and colorectal cancer. We have also come to realise that increasing the cure rate by $5 \%$ in very common diseases is of major public health importance. We have become interested in small increments of progress and want to detect them.

In the early days of clinical trials in NSCLC there was a rather unsophisticated approach towards the chemotherapy used in these studies since it was assumed that all cytotoxic drugs were more or less equal. Furthermore, really active regimens had not then been defined. In the last 15 years refinement of combination chemotherapy in advanced disease has led to the identification of new regimens in which responses can be seen in $25-50 \%$ of patients with metastatic or locally advanced disease and even some complete responses. These combinations have often been based on cisplatin combination chemotherapy with the addition of drugs such as mitomycin, ifosfamide, and etoposide. At the present time the most active regimens appear to be a combination of mitomycin, vinblastine and cisplatin, and mitomycin, ifosfamide and cisplatin. ${ }^{5}$ Other changes in the methodology of trials in the UK have included the advent of modern staging techniques with a much better description of patient categories, agreed response criteria, and improved methods of assessment.

These shifting sands have meant that analysis of the results of trials of chemotherapy in all stages of the disease have led to very conflicting results. Nevertheless, it might reasonably be argued that, if one compares the use of chemotherapy against untreated controls, however much these other factors might alter the magnitude of the observed effect, the effect ought to be in the same direction - that is, qualitatively similar.

It is precisely in this situation that an overview analysis of randomised trials is so helpful. If such analyses are to be undertaken they should not be based on published trials identified by literature surveys. Publication bias leads to a distorted result with no precise estimate of any difference. The most reliable analysis is based on the original patient data of all the trials which have ever been conducted, whether published or not. Such a laborious undertaking has recently been completed by the Medical Research Council and the Institut Gustave Roussy and the findings have recently been published. ${ }^{6}$

At the outset the collaborating groups made a clear distinction between chemotherapy in the "modern" era and chemotherapy which had been given in the late 1960 s and early 1970s, using the inclusion of cisplatin in the regimen to identify the "modern" combinations. This did not result from a belief that cisplatin was a unique drug in these combinations, but was simply a means of identifying regimens which one might regard as recent. This cannot be done simply on the basis of the date at which the trial was started, since many trials are conducted over several years. The different clinical settings in which chemotherapy has been compared with control groups were subdivided according to the primary treatment. Thus, there were comparisons between (1) chemotherapy and control in patients undergoing surgical resection, (2) radical radiotherapy for inoperable disease, (3) combinations of surgery and radical radiotherapy, and (4) palliative treatment for metastatic disease.

Somewhat to our surprise the results have suggested that, in three of these four categories, chemotherapy of the "modern" type confers a survival advantage. In the surgical comparison this is approximately $5 \%$ at five years, in patients treated with radical radiotherapy for a locally advanced disease it is $1-4 \%$ at five years, and in best supportive care it is $10 \%$ at one year. The estimates of the differences are extremely stable since many thousands of patients have been included in the analysis. There were 4357 patients in the surgical comparison, 3033 in the radical radiotherapy group, and 1190 in the best supportive care trials. In the trial where patients were treated with surgery and radical radiotherapy with or without chemotherapy the result was inconclusive. Only 807 patients were 
available in this comparison and, for this reason, the result lacks precision.

A remarkable consequence of the subdivision of the chemotherapy, and one which was not anticipated, was that an adverse effect of chemotherapy was seen in early trials where single agent continuous alkylating agents were used, usually cyclophosphamide or a nitrosourea. We do not know what increased the death rate in these trials since the data are too old to find the causes of death. This does not prove that all alkylating agent therapy is harmful but that long term continuous oral alkylating agent therapy is deleterious.

Does this overview analysis settle the issue as far as chemotherapy is concerned? It does not for several reasons. Firstly, there is considerable heterogeneity in the trials and the result gives an overall estimate from widely differing studies. The result from currently used chemotherapy may or may not be better than this. Secondly, none of these studies contained analyses of either economics or quality of life. Many clinicians would find a survival difference of a few percent not very compelling if achieved only at the expense of toxicity and considerable economic and organisational cost.

We have very few data on the quality of life of patients undergoing this form of treatment. Such data are now being collected in some studies and earlier results suggest that chemotherapy controls symptoms including cough, breathlessness, and pain, and increases well being. This is perhaps not surprising since there have also been great advances in the control of chemotherapy induced nausea and vomiting in recent years. Interestingly, some of the most positive studies in the meta analysis have only used two cycles of chemotherapy and the optimum duration of chemotherapy is entirely unknown.

A concerted effort is now needed in the UK to design some very large scale pragmatic trials to determine whether straightforward regimens of chemotherapy can easily be administered to patients and whether they improve survival. Large numbers of patients are needed in these trials because the survival advantage is likely to be small. Such studies need to be combined with some economic assessment during the trial. The cost of three cycles of platinum based chemotherapy will probably be in the order of $£ 500$ to
$£ 1000$, depending on whether the patient receives the chemotherapy as a day case or with an overnight stay in hospital. If one in 20 patients was cured by such a treatment the cost per life saved would be $£ 10000$ to $£ 20000$. This is a very low figure in comparison with many medical interventions.

It would be a big step forward if we could change the climate of pulmonary oncology in the UK so that large scale pragmatic studies become part of the general culture of lung cancer treatment. To do this we need the enthusiastic collaboration of chest physicians and oncologists. There are many exciting questions to come. New drugs are now available or are immediately on the horizon such as navelbine, gemcitabine, and taxol derivatives. ${ }^{7-9}$ If we can show that chemotherapy is truly worthwhile, there are several other questions which will follow and which will need to be addressed in large studies. Furthermore, the economic benefit of treatment and the impact of treatment on quality of life are best assessed in the rigorous setting of randomised, large scale, clinical trials.

Department of Oncology,

University College London School of Medicine,

London W1P 8BT, UK

R L SOUHAMI

1 Bleehen NM, Girling DJ, Machin D, Stephens RJ. A Medical Research Council (MRC) randomised trial of palliative radiotherapy with two fractions or a single fraction in patients with inoperable non-small-cell lung cancer (NSCLC) and poor performance. Br f Cancer 1992;65:934-41.

2 Le Chevalier T, Arriagada R, Quoix E, Ruffie P, Martin M, Tarayre M, et al. Radiotherapy alone versus combined chemotherapy and radiotherapy in nonresectable non-small-cell lung cancer: first analysis of a randomized trial in 353 patients. $\mathcal{F}$ Natl Cancer Inst 1991;83:417-23.

3 Pisters KMW, Kris MG, Gralla RJ, Zaman MB, Heelan RT, Martini N. Pathologic complete response in advanced non-small-cell lung cancer following preoperative chemotherapy: implications for the design of future non-small-cell lung cancer combined modality trials. $\mathcal{F}$ Clin Oncol 1993; 11:1757-62.

4 Davis DL, Hoel D, Fox J, Lopez A. Epidemiology: international trends in cancer mortality in France, West Germany, Italy, Japan, England and cancer mortality in France, West Germany,
Wales, and the USA. Lancet 1990;336:474-81.

5 Currie DC, Miles DW, Drake JS, Rudd R, Spiro SG, Earl HM, et al. Mitomycin, ifosfamide and cisplatin in non-small cell lung cancer. Cancer Chemother Pharmacol 1990;25:380-1.

6 Non-Small Cell Lung Cancer Collaborative Group. Chemotherapy in nonsmall cell lung cancer: a meta-analysis using updated data on individual patients from 52 randomised clinical trials. BMF 1995;311:899-909.

7 Le Chevalier T, Brisgand D, Douillard J-Y, et al. Randomized study of vinorelbine and cisplatin versus vindesine and cisplatin versus vinorelbine alone in advanced non-small-cell lung cancer: results of a European alone in advanced non-small-cell lung cancer: results of a Europ
multicenter trial including 612 patients. $f$ Clin Oncol 1994;12:360-7.

8 Rowinsky EK, Donehower RC. Paclitaxel (taxol). N Engl f Med 1995;332: 1004-14.

9 Lilenbaum RC, Green MR. Novel chemotherapeutic agents in the treatment of non-small-cell lung cancer. $\mathcal{F}$ Clin Oncol 1993;11:1391-402. 